\title{
Development and Performance Evaluation of Fine Stage for 3-DOF Error Compensation
}

\author{
Jae-Chang Lee ${ }^{1}$, Min-Jae Lee ${ }^{1}$, Hoon-Hee Lee ${ }^{1}$ and Seung-Han Yang ${ }^{1, *}$ \\ ${ }^{1}$ School of Mechanical Engineering, Kyungpook National University, 80, Daehak-ro, Buk-gu, Daegu, South Korea, 41566 \\ *Corresponding author
}

\begin{abstract}
In this paper, a fine stage based on parallel mechanism was developed for 3-DOF error compensation to improve the form accuracy of the workpiece. This mechanism with 3-DOF motions in the plane was designed as the parallel structure for minimization and high structural stiffness. The static and dynamic stiffness analysis of the developed flexure-based mechanism have been conducted to verify the validity in displacement of workspace in the $\mathrm{X}$ - and $\mathrm{Y}$ - directions. For accurate positioning, the effect by Abbe's offset between the measuring and driving coordinate system was analyzed and minimized. The displacement of piezoelectric actuators was determined from the measured data and inverse kinematic. The performance of the developed fine stage was evaluated through the basic performance evaluation.
\end{abstract}

Keywords-form accuracy; fine stage; parallel mechanism; error compensation

\section{INTRODUCTION}

The high-accuracy miniature components with sub-micro meter level are increasingly in demand for high tech-industries such as electro-optic, automotive, biotechnology, information technology etc [1-3]. Ultra-precision machine technology have the advantages of being able to manufacture geometrically complex miniature components to high accuracy [4]. To realize the ultra-precision machining technology, the improvement of the positioning accuracy of the machine tool is essential work. Recent studies on dual-stage feed drive (DSFD) systems have been conducted to achieve high precision positioning. A dualstage feed drive provides the capacity of a large workspace with the property of high precision motion [5]. But, various errors affect the positioning accuracy of the machine tool and occur undesirable multi-degree-of-freedom motions. These motions eventually distort the relative position and orientation between the workpiece and tool. Eventually, undesirable mutidegree-of-freedom directly affect the form accuracy of the workpiece and deteriorate the quality of the components.

Errors to cause multi-degree-of-freedom can be generally classified into static errors, quasi-static errors and dynamic errors [6,7]. The basic inaccuracy of the machine tool is due to the geometric error and it accounts for $70 \%$ of the total error [8]. So, the measurement and compensation technology into the geometric errors are necessary for the positioning accuracy of the machine tool.

Various studies have been conducted into measurement and compensation for these errors. Lee proposed five-DOF motion measurement system to measure geometric errors of linear axes and designed optimal measurement system to minimize the standard uncertainty of measured geometric errors [9]. Lee suggested algorithm for accurately measuring the squareness error of linear axes using laser interferometer. The Abbe's offset caused by optics setup can be eliminated by the algorithm [10].

Also, a dual-stage feed drive system is used to compensate errors of linear axes. Lee proposed the two stages simultaneously controlled as a dual servo system to achieve positioning within 20 nanometers in planar motion. The two stages are consisted of the global stage configured with three linear motors and the micro stage by activating three PZT actuators [11]. Yang developed a precision positioning system based on macro-micro dual-drive in order to achieve highspeed and ultra-precision positioning. This system is composed of the macro stage as a linear motor drive and the micro stage as the PZT actuator and flexure hinge [12].

However, most systems are difficult to apply machining because the structural limit and the performance evaluation of the suggested system was not proceeded from the machining results.

In this study, a fine stage was developed to improve the form accuracy of the workpiece. The suggested stage improves the positioning accuracy by measuring and compensating undesirable 3-DOF motion of a linear-axis in feed direction when machining. The developed planar fine stage was described in Section 2. The 3-DOF error compensation system was explained for undesirable 3-DOF motion in Section 3. The performance of the compensation system was evaluated through the basic performance evaluation in Section 4. Finally, the conclusions of this study are presented.

\section{DeVelopment of the Planar Fine Stage}

\section{A. The Structure of a 3-DOF Parallel Mechanism}

The suggested planar fine stage is a structure of a 3-DOF parallel mechanism. The developed 3-DOF parallel mechanism is consisted of three piezoelectric actuators and joints based on flexure hinge. The parallel mechanism is designed for miniaturization of the system and high structural stiffness. Also, the joints based on flexure hinge are utilized for positioning accuracy in micro-area. The piezoelectric actuators are used for reproducibility of the position and fast response time. The fine state has 3-DOF motions to be measured and compensated in the feed direction. The parallel mechanism is composed of the 
base platform, moving platform and six revolute joints based on the circular notch hinge as shown in figure 1.

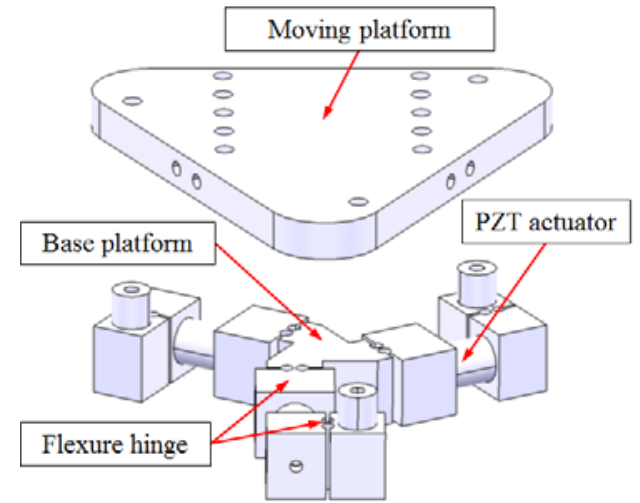

FIGURE I.

THE STRUCTURE OF A PARLLEL MECHANISM

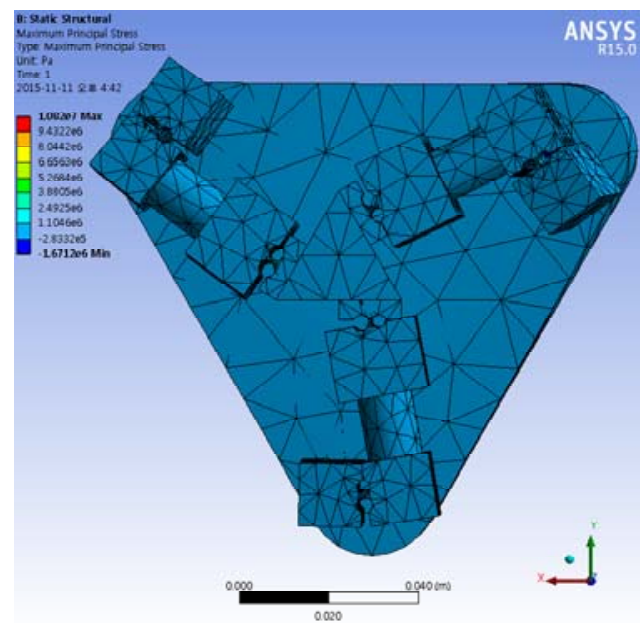

FIGURE II.

STATIC ANALYSIS OF THE FINE STAGE

\section{B. Static Analysis of the Fine Stage}

The developed fine stage is required for the implementation of three identical piezoelectric actuators. The fine stage was made of Aluminum 6061. The fine state based on flexure hinge was analyzed statically using FE analysis to check on the maximum stress value and the uniformity in displacement of workspace in the $\mathrm{X}$ and $\mathrm{Y}$ directions. Figure 2 demonstrates the stress distribution and deflections due to a fixed piezoelectric actuator displacement of 15 microns. The stress reached a maximum of $16 \mathrm{MPa}$ which is $9.4 \%$ of the material yield strength. Table 1 shows the finite element analysis parameters.

TABLE I. THE FINITE ELEMET ANALYSIS PARAMETERS

\begin{tabular}{|l|l|}
\hline \multicolumn{1}{|c|}{ Parameter } & \multicolumn{1}{c|}{ Value } \\
\hline Part material & Aluminum 6061 \\
\hline Material young modulus & $70 \mathrm{GPa}$ \\
\hline Material yield strength & $170 \mathrm{MPa}$ \\
\hline Poisson ratio & 0.33 \\
\hline Maximum nodal stress & $16 \mathrm{MPa}$ \\
\hline
\end{tabular}

\section{Dynamic Analysis of the Fine Stage}

The dynamic FE analysis was required to get an estimate of the natural frequencies and mode shapes of the fine stage within the range of the operating frequency. The operating frequency range was selected to be from 0 to $1000 \mathrm{~Hz}$. This range represents the frequencies that might be excited with a milling process $1000 \mathrm{rpm}$ spindle and two teeth end mill [13].

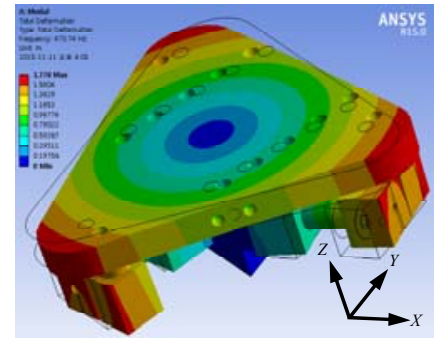

(a) First mode shape at $470.7 \mathrm{~Hz}$.

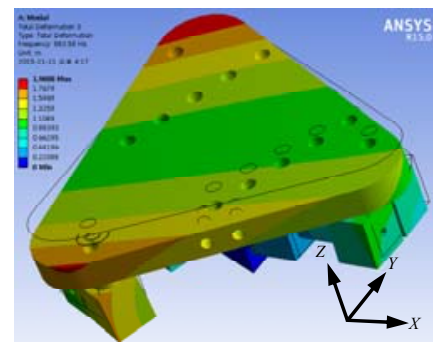

(c) Third mode shape at $883.6 \mathrm{~Hz}$.

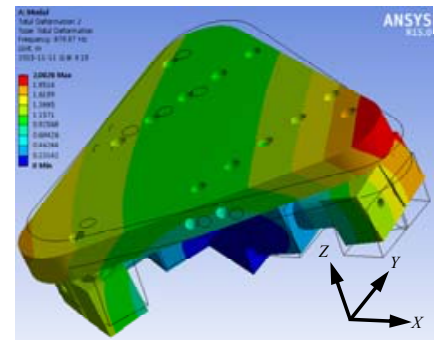

(b) Second mode shape at $879.9 \mathrm{~Hz}$.

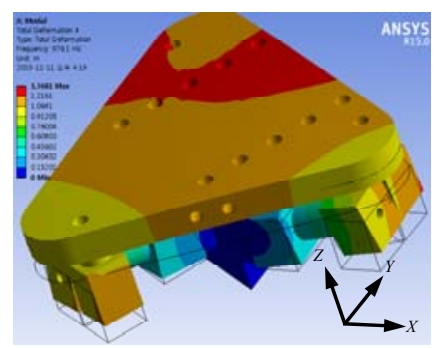

(d) Fourth mode shape at $976.1 \mathrm{~Hz}$.

\section{FIGURE III. THE MODE SHAPES OF THE FINE STAGE}

Figure 3 illustrate the shapes in the range of 0 to $1000 \mathrm{~Hz}$. There are four natural frequencies in the selected working frequency range with a first natural frequency of $470.7 \mathrm{~Hz}$. This is the most flexible mode. These four mode shapes are located away from the piezoelectric actuator controller bandwidth but can be excited by a cutting process. The spindle speed and the number of teeth of the milling cutter must be selected to avoid these frequencies.

\section{THE 3-DOF ERROR COMPENSATION SYSTEM}

This fine stage is fixed to a linear-axis and transferred by the command value of a linear-axis. And the capacitive sensors and reference mirror are used to measure and compensate 3DOF errors of a linear axis. The capacitive sensors are fastened to the moving platform and the reference mirror is installed on the external to minimize the standard uncertainty of the measured error.

\section{A. 3-DOF Error Measurement}

The reference coordinate system $\{\boldsymbol{F}\}$ corresponds to the workpiece coordinate system $\{\boldsymbol{P}\}$ to be set at the position that occurs in machining. The coordinate system has a relative position and orientation between the coordinate system of the tool and workpiece coordinate system caused by multi-DOF motion to take place due to various errors of a linear-axis as show in figure 4 . The three capacitive sensors and reference mirror are utilized for measurement of 3-DOF error of a linear 
axis. But, Abbe's offset ( $\mathrm{o}_{\mathrm{x}}, \mathrm{o}_{\mathrm{y}}, \mathrm{o}_{\mathrm{z}}$ ) among the coordinate systems arise owing to discordance between the reference coordinate system $\{\boldsymbol{F}\}$ and measurement coordinate system $\{\boldsymbol{M}\}$ by the structural limit. The clear relation definition between the coordinate systems is necessary because 3-DOF motion is defined in the measurement coordinate system $\{\boldsymbol{M}\}$. The Abbe's offset between the coordinate systems has an adverse effect on the positioning motion to compensate the reference coordinate system. In particular, the 1-DOF positioning motion to compensate is affected by the roll motion in the feed direction ( $Y$-axis) as shown in figure 4. So, the roll motion is measured by additional one capacitive sensor to minimize the effect of the Abbe's offset. 2-DOF motion to compensate in the coordinate system $\{\boldsymbol{F}\}$ using the measured 3DOF motion is the same as equation (1). Here, $\delta_{x y}$ presents a straightness error in the Y-axis direction defined by the coordinate system $\{\boldsymbol{F}\}$ and $\varepsilon_{z y}$ means a rotation error of the Zaxis direction. Similarly, $\delta_{x y, M}$ signifies a straightness error in the $\mathrm{Y}$-axis direction defined by the coordinate system $\{\boldsymbol{M}\}$. And, $o_{z}$ refers to the Abbe's offset in the Z-axis direction between the coordinate systems.

$$
\begin{aligned}
& \delta_{x y}=\delta_{x y, M}+\varepsilon_{y y, M} \times o z \\
& \varepsilon_{z y}=\varepsilon_{z y, M}
\end{aligned}
$$

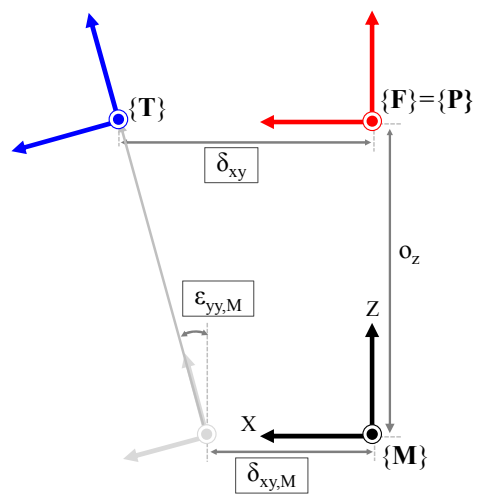

FIGURE IV. ABBE'S OFFSET BETWEEN REFERENCE COORDINATE SYSTEM AND DRIVING COORDINATE SYSTEM

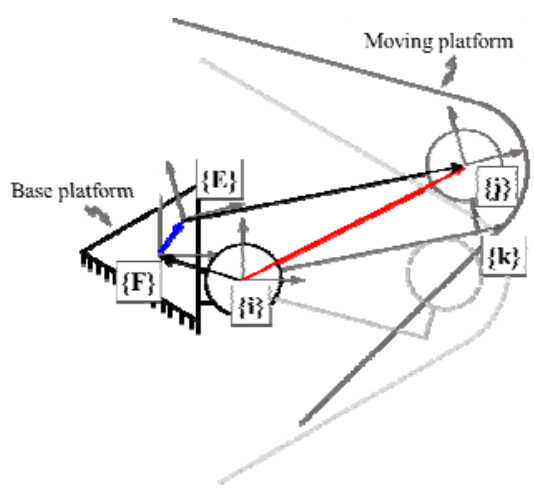

FIGURE V.

MODELING OF COORDINATE SYSTEM IN 3-DOF PARALLEL MECHANISM

\section{B. Modeling of Coordinate System for Kinematic Analysis}

The displacement of each piezoelectric actuators is calculated by using the inverse kinematic to realize 3-DOF error compensation. Figure 5 shows the coordinate systems in each of the joints and piezoelectric actuators. The coordinate system $\{\boldsymbol{E}\}$ is located at the center of the moving platform with random position and orientation. And the coordinate system $\{\boldsymbol{i}\}$, $\{\boldsymbol{j}\}$ are the joint coordinate system connected by each of the base platform and moving platform. And also, the coordinate system $\{\boldsymbol{k}\}$ presents the link coordinate system. Equation (2) is the relationship between the coordinate systems by the kinematic chain. Here, $\boldsymbol{\tau}_{i}^{j}$ denotes a position and orientation of the coordinate system $\{\boldsymbol{j}\}$ defined in the coordinate system $\{\boldsymbol{i}\}$. $\boldsymbol{P}_{i}{ }^{j}$ is described as the relative position vector between the origin coordinate system $\{\boldsymbol{i}\},\{\boldsymbol{j}\}$. The position and orientation of each joint coordinate system $\{\boldsymbol{i}\},\{\boldsymbol{j}\}$ are the nominal value as the design variables. $\boldsymbol{\tau}_{F}^{E}$ is presented in the 3-DOF error compensation as known values in the reference coordinate system $\{\boldsymbol{F}\}$. The relationship of the coordinate system $\{\boldsymbol{i}\},\{\boldsymbol{j}\}$ using the equation (2) to calculate the displacement of piezoelectric actuators is determined as shown in the equation (3). The displacement $l_{i}(\mathrm{i}=1,2,3)$ of piezoelectric actuators according to compensate the values using the relative position vector between the coordinate system is calculated as show in equation (4).

$$
\boldsymbol{\tau}_{F}^{i} \boldsymbol{\tau}_{i}^{j}=\boldsymbol{\tau}_{F}^{E} \boldsymbol{\tau}_{E}^{j}
$$

where,

$$
\begin{aligned}
& \boldsymbol{\tau}_{F}^{i}=\left[\begin{array}{cc}
\mathbf{I} & \mathbf{P}_{F}^{i} \\
\mathbf{0} & 1
\end{array}\right] \\
& \boldsymbol{\tau}_{i}^{j}=\left[\begin{array}{cc}
\mathbf{R} & \mathbf{P}_{i}^{j} \\
\mathbf{0} & 1
\end{array}\right] \\
& \boldsymbol{\tau}_{F}^{E}=\left[\begin{array}{ll}
\mathbf{R} & \mathbf{T} \\
\mathbf{0} & 1
\end{array}\right] \\
& \boldsymbol{\tau}_{E}^{j}=\left[\begin{array}{cc}
\mathbf{I} & \mathbf{P}_{E}^{j} \\
\mathbf{0} & 1
\end{array}\right] \\
& \mathbf{I}=\left[\begin{array}{lll}
1 & 0 & 0 \\
0 & 1 & 0 \\
0 & 0 & 1
\end{array}\right] \\
& \mathbf{R}=\left[\begin{array}{ccc}
1 & -\varepsilon_{z y} & 0 \\
\varepsilon_{z y} & 1 & 0 \\
0 & 0 & 1
\end{array}\right] \\
& \mathbf{0}=\left[\begin{array}{lll}
0 & 0 & 0
\end{array}\right] \\
& \mathbf{T}=\left[\begin{array}{lll}
\delta_{x y} & \delta_{y y} & 0
\end{array}\right]^{T} \\
& \boldsymbol{\tau}_{i}^{k}=\left(\boldsymbol{\tau}_{R}^{i}\right)^{-1} \boldsymbol{\tau}_{R}^{E} \boldsymbol{\tau}_{E}^{j}\left(\boldsymbol{\tau}_{k}^{j}\right)^{-1} \\
& l_{\mathrm{i}}=\left|\mathbf{P}_{i}^{j}-\mathbf{P}_{k}^{j}\right|-l_{0} \\
& l_{0}=\text { Initial length of PZT actuator }
\end{aligned}
$$




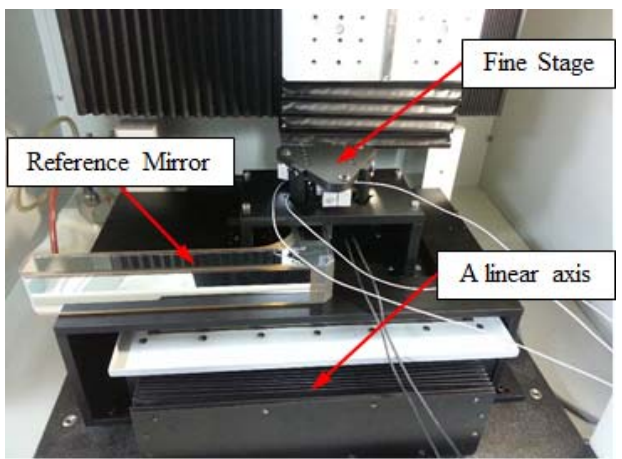

FIGURE VI. EXPERIMENTAL SETUP AT 3-AXIS MACHINE TOOL

\section{EXPERIMENTAL PERFORMANCE EVALUATION}

The experiment proceeded with 3 -axis machine tool using the developed fine stage and analyzed the results. The capacitive sensors (4810 module, 2812 probe, ADE Technology Inc., U. S. A.), reference mirror and PZT actuators(P-840.10, PI GmbH, Germany)were used in the experiment. Figure 6 shows the experimental setup. The fine stage was fixed to a linear axis and the reference mirror was located outside the machine tool. The stage compensated 3-DOF errors (horizontal straightness error of $X$-axis, linear displacement error of $Y$-axis and Yaw error of Z-axis) in the feed direction of the $\mathrm{Y}$-axis. The step response was evaluated to verify the motion implementation and performance of the developed fine stage. The step motion of $20 \mathrm{~nm}, 0.2 \operatorname{arcsec}$ was realized to $1-\mathrm{DOF}$ positional and rotational motion implementation. Figure 7 demonstrates the clear step form of the measurement result.
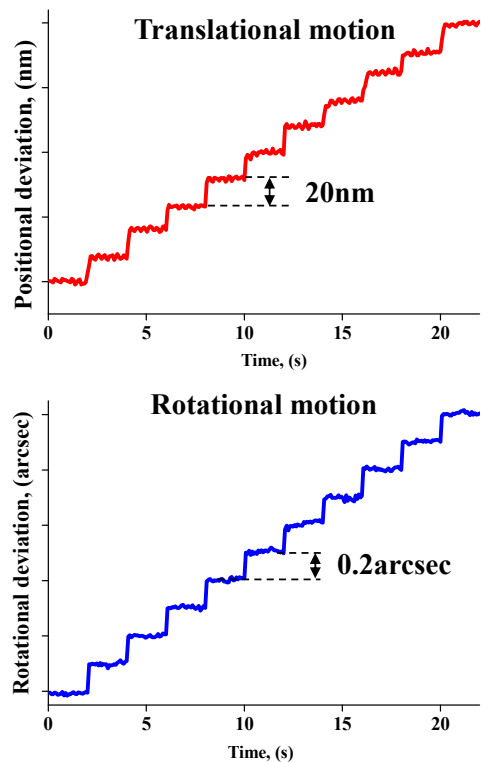

FIGURE VII.

STEP RESPONSE FOR POSITIONAL AND ROTATIONAL MOTIONS

\section{CONCLUSION}

The fine stage with 3-DOF error compensation is proposed for the quality improvement of the workpiece. This stage improves the form accuracy of the workpiece by compensation about undesirable 3-DOF motion of a linear-axis in feed direction when machining. The influence on Abbe's error is minimized for accurate compensation considering the Abbe's offset between the coordinate systems. The motion implementation and performance of the developed fine stage was evaluated by the step response.

\section{ACKNOWLEDGMENT}

This work was supported by the National Research Foundation of Korea (NRF) grant funded by the Ministry of Education (2010-0020089) and the Korea Government (MSIP) (2013R1A2A2A01067917).

\section{REFERENCES}

[1] X. Luo, K. Cheng, D. Webb, and F. Wardle, "Design of ultraprecisoin machine tools with applications to manufacture of miniature and micro components," J. Mater. Process. Technol., vol. 167, pp. 515-528, 2005.

[2] S. J. Zhang, S. To, S. J. Wang, and Z. W. Zhu, "A review of surface roughness gerneration in ultra-precision machining," Int. J. Mach. Tool. Manu., vol. 91, pp. 76-95, 2015.

[3] K. F. Ehmann, D. Bourell, M. L. Culpepper, T. J. Hodgson, T. R. Kurfess, M. Madou, K. Rajurkar, and R. E. DeVor, "International assessment of research and development in micromanufacturing," World Technology Evaluation Center, Baltimore, Maryland, 2005

[4] S. Filiz, C. M. Conley, M. B. Wasserman, and O. B. Ozdoganlar, "An experimental investigation of micro-machinability of copper 101 using tungsten carbide micro-endmills," Int. J. Mach. Tool. Manu., vol. 47, pp. 1088-1100, 2007.

[5] A. T. Elfizy, G. M. Bone, and M. A. Elbestawi, "Design and control of a dual-stage feed drive,” Int. J. Mach. Tool. Manu., vol. 45, pp. 153-165, 2015.

[6] H. Schwenke, W. Knapp, H. Haitjema, A. Weckenmann, R. Schmitt, and F. Delbressine, "Geometric error measurement and compensation of machines-An update," CIRP Annals - Manufacturing Technology, vol. 57, pp. 660-675, 2008.

[7] J. C. Lee, K. I. Lee, and S. H. Yang, "Performance evaluation of fiveDOF motion under static and dynamic conditions of ultra-precision linear stage," J. Korean Soc. Precis. Eng., vol. 31, pp. 423-430, May 2014.

[8] R. Ramesh, M. A. Mannan, and A. N. Poo, "Error compensation in machine tools - a review Part I: geometric, cutting-force induced and fixure-dependent errors," Int. J. Mach. Tool. Manu., vol. 40, pp. 12351256, January 2000.

[9] K. I. Lee, J. C. Lee, and S. H. Yang, "The optimal design of a measurement system to mearsure the geometric errors of linear axes," Int. J. Adv. Manuf. Technol., vol. 66, pp. 141-149, 2013.

[10] D. M. Lee, H. H. Lee, and S. H. Yang, "Analysis of the squareness measurement using laser interferometer," J. Korean Soc. Precis. Eng., vol. 29, pp. 863-872, 2012.

[11] C. W. Lee, and S. W. Kim, "An ultraprecision stage for alignment of wafers in advanced microlithography," Precision engineering, vol. 21, pp. 113-122, 1997

[12] Q. Xu, "Design and testing of a novel XY micropositioning stage with dual ranges and resolutions," IEEE International conference on robotics \& automation (ICRA) China, pp. 2351-2356, 2014.

[13] Wit Grzesik, Advanced Machining Processes of Metallic Materials: Theory, Modelling and Applications, 1st ed., Oxford: Great Briain, 2008 , pp. $120-122$. 